Ki net i cs of NADP+/NADPH reduct $i$ on oxi dat $i$ on cat al yzed by the ferredoxi $n-N A D(P)+$ reduct ase from the green sulfur bact eri um Chl or obacul um tepi dum

\begin{tabular}{|l|l|}
\hline 著者 & $\begin{array}{l}\text { Seo Dai suke, Ki tashi na Nasahar u, Sakur ai } \\
\text { Takeshi, I noue Kazuhi to }\end{array}$ \\
\hline $\begin{array}{l}\text { j our nal or } \\
\text { publ i cat i on t i t l e }\end{array}$ & Phot osynt hesi s Resear ch \\
\hline vol une & 130 \\
\hline nunber & $1-3$ \\
\hline page $r$ ange & $479-489$ \\
\hline year & $2016-12-01$ \\
\hline URL & ht t p: //hdl . handl e. net /2297/45922 \\
\hline
\end{tabular}




\title{
Kinetics of $\mathrm{NADP}^{+} / \mathrm{NADPH}$ reduction-oxidation catalyzed by the ferredoxin-NAD(P) ${ }^{+}$ reductase from the green sulfur bacterium Chlorobaculum tepidum
}

\author{
Daisuke Seo ${ }^{1 *}$, Masaharu Kitashima ${ }^{2,3}$, Takeshi Sakurai ${ }^{1}$, Kazuhito Inoue ${ }^{2,3}$ \\ ${ }^{1}$ Division of Material Science, Graduate School of Natural Science and Technology, Kanazawa \\ University, Kakuma, Kanazawa, Ishikawa 920-1192, Japan \\ ${ }^{2}$ Department of Biological Sciences and ${ }^{3}$ Research Institute for Integrated Science, Kanagawa \\ University, Tsuchiya, Hiratsuka, Kanagawa 259-1293, Japan \\ *To whom correspondence should be addressed: \\ Daisuke Seo, Division of Material Science, Graduate School of Natural Science and Technology, \\ Kanazawa University, Kakuma, Kanazawa, Ishikawa 920-1192, Japan \\ Tel: +81-76-264-5683 \\ Fax: +81-76-264-5742 \\ Email: dseo@se.kanazawa-u.ac.jp
}

\section{Keywords}

Flavoenzyme; Adrenodoxin; Putidaredoxin; Stopped flow; Charge transfer complex, Kinetic isotope effect

\section{Abbreviations}

A, absorbance; Ad, adrenodoxin; AdR, adrenodoxin reductase; Bs, Bacillus subtilis; Ct, Chlorobaculum tepidum; CT, charge transfer; CTC, charge transfer complex; Ec, Escherichia coli; FAD, flavin adenine dinucleotide; Fd, ferredoxin; FNR, ferredoxin-NAD(P) ${ }^{+}$oxidoreductase; GR, glutathione reductase; HEPES, 2-[4-(2-hydroxyethyl)-1-piperazinyl]ethanesulfonic acid; NADPD, $\left(4 S-{ }^{2} \mathrm{H}\right)$-reduced nicotinamide adenine dinucleotide phosphate; Pd, putidaredoxin; PdR, putidaredoxin reductase; TrxR, bacterial NADPH-thioredoxin reductase;

Suffixes: ox, oxidized; red, reduced.

\section{Acknowledgements}

We thank Dr. Hidehiro Sakurai for stimulating discussions. This work was supported in part by a grantin-aid for Scientific Research on Innovative Areas (No. 24107004) and the Strategic Research Base Development Program for Private Universities from the Ministry of Education, Culture, Sports, Science and Technology (MEXT), Japan (to KI). 


\begin{abstract}
Ferredoxin-NAD(P) ${ }^{+}$oxidoreductase (FNR, [EC 1.18.1.2], [EC 1.18.1.3]) from the green sulfur bacterium Chlorobaculum tepidum $(C t \mathrm{FNR})$ is a homodimeric flavoprotein with significant structural homology to bacterial NADPH-thioredoxin reductases. CtFNR homologs have been found in many bacteria, but only in green sulfur bacteria among photoautotrophs. In this work, we examined the reactions of $C t \mathrm{FNR}$ with $\mathrm{NADP}^{+}$, NADPH and $\left(4 S-{ }^{2} \mathrm{H}\right)-\mathrm{NADPD}$ by stopped-flow spectrophotometry. Mixing $C t \mathrm{FNR}_{0 \mathrm{x}}$ with NADPH yielded a rapid decrease of the absorbance in flavin band I centered at $460 \mathrm{~nm}$ within $1 \mathrm{~ms}$, then the absorbance further decreased gradually. The magnitude of the decrease increased with increasing NADPH concentration, but even with 50 -fold molar excess NADPH, the absorbance change was only $\sim 45 \%$ of that expected for fully reduced protein. The absorbance in the charge transfer (CT) band centered around $600 \mathrm{~nm}$ increased rapidly within $1 \mathrm{~ms}$, then slowly decreased to about $70 \%$ of the maximum. When $C t \mathrm{FNR}_{\text {red }}$ was mixed with excess $\mathrm{NADP}^{+}$, the absorbance in the flavin band I increased to about $70 \%$ of that of $C t \mathrm{FNR}_{\text {ox }}$ with an apparent rate of $\sim 4 \mathrm{~s}^{-1}$, whereas almost no absorption changes were observed in the CT band. Obtained data suggest that the reaction between $C t \mathrm{FNR}$ and $\mathrm{NADP}^{+} / \mathrm{NADPH}$ is reversible, in accordance with its physiological function.
\end{abstract}




\section{Introduction}

Ferredoxin-NAD $(\mathrm{P})^{+}$reductase ([EC 1.18.1.2], [1.18.1.3], FNR) is a member of the NAD $(\mathrm{P}) \mathrm{H}-$ dependent oxidoreductases of the flavoprotein superfamily containing a non-covalently bound flavin adenine dinucleotide (FAD) or flavin mononucleotide (FMN) as a prosthetic group. FNR can reversibly catalyze either one- and two-redox-equivalent transfer with the small iron-sulfur protein ferredoxin ( $\mathrm{Fd}$ ) and $\mathrm{NAD}(\mathrm{P})^{+} / \mathrm{NAD}(\mathrm{P}) \mathrm{H}$, respectively, using the redox properties of the flavin prosthetic group. In the final step of the electron transport chain in photosynthesis, Fd and FNR mediate electron transfer from a type-I photosynthetic reaction center to $\mathrm{NAD}(\mathrm{P})^{+}$(Knaff and Hirasawa 1991; Sétif 2001; Seo and Sakurai 2002). In non-photosynthetic processes, Fd as well as small iron sulfur proteins, adrenodoxin (Ad) and putidaredoxin (Pd) participate in electron transfer in reactions involved in synthesis of a wide range of cellular materials such as fatty acid desaturation (cytochrome P450), nitrite reduction, sulfite reduction, and $S$-adenosylmethionine radical-dependent reactions in DNA and coenzyme synthesis (Munro et al. 2007; Knaff and Hirasawa 1991; Bianchi et al. 1993; Wan and Jarrett 2002). In the latter processes, FNR, adrenodoxin reductase (AdR, [EC 1.18.1.6]) and putidaredoxin reductase (PdR, [EC 1.18.1.5]) catalyze the reduction of Fd, Ad and Pd by NAD(P)H. Thus the redox coupling of Fd-FNR-NAD(P) $)^{+} / \mathrm{NAD}(\mathrm{P}) \mathrm{H}$ constitutes a key process of a widespread redox network in living organisms. The physiological direction of the process is assumed to be related to the redox midpoint potentials of the reaction partner(s).

Each of FNR, AdR and PdR has two nucleotide-binding domains, one for FAD/FMN and the other for $\mathrm{NAD}(\mathrm{P})^{+} / \mathrm{H}$ (Correll et al. 1993; Ziegler et al. 1999; Sevrioukova et al. 2004; Muraki et al 2010; Medina and Gomesz-Moreno 2004; Alverti et al 2008). This protein topology is typical of flavin-containing $\mathrm{NAD}(\mathrm{P}) \mathrm{H}$-dependent oxidoreductases including disulfide reductases (Dym and Eisenberg 2001; Alverti et al. 2008). Based on structural and phylogenetic information, FNRs, PdR and AdR are classified into two major groups of the flavoprotein superfamily, namely the plant-type and the glutathione reductase (GR)-type FNR superfamilies and further divided into several subgroups (Alverti et al. 2008; Ceccarelli et al. 2004; Seo et al. 2004). Among FNRs, the bacterial NADPHthioredoxin reductase-type (TrxR-type) FNR, a subgroup of the GR-type FNR superfamily, is unique in their homo-dimeric form with high structural homology to Escherichia coli TrxR (EcTrxR) (Muraki et al. 2010; Komori et al. 2010; Waksman et al. 1994). TrxR-type FNR and its homologs have been found in bacteria and archaea, including green sulfur bacteria, Firmicutes, several $\alpha$-proteobacteria including Rhodospirillaceae and the archaeal genus Sulfolobus (Seo et al. 2002, 2004, 2009; Yan et al. 2014; Mandai et al. 2009), but it was not found in eukaryotes (Kyoto Encyclopedia of Genes and Genomes, Kanehisa et al. 2016). Among members of the TrxR-type FNR subfamily, the protein from the photoautotrophic green sulfur bacterium Chlorobaculum tepidum (formerly Chlorobium tepidum; $C t \mathrm{FNR}$ ) has been isolated as a component required for $\operatorname{NAD}(\mathrm{P})^{+}$photoreduction (Seo and Sakurai 2002). C. tepidum carries out non-oxygenic photosynthesis using various sulfur compounds as the 
electron sources (Ogawa et al. 2008; Sakurai et al. 2010) and has a type-I photosynthetic reaction center that can reduce Fds directly (Kjær and Sheller 1996; Seo et al. 2001; Tsukatani et al. 2004). In vitro, purified $C t \mathrm{FNR}$ can catalyze the reduction of $\mathrm{NAD}(\mathrm{P})^{+}$in the presence of the photosynthetic reaction center and Fd from C. tepidum (Seo and Sakurai 2002). The unique features of the green sulfur bacteria among phototrophs are that they use the reductive TCA cycle as the main carbon assimilation pathway (Buchanan and Arnon1990; Tang et al. 2010) which have been found among several bacterial groups (Hügler and Sievert 2011), and that FNR and Fds are phylogenetically unrelated to their counterparts in oxygenic photosynthetic organisms/organelles but related to those from several bacterial groups described above. These would provide useful information on the origin and evolution of photosynthesis.

Although the redox reaction catalyzed by FNR is theoretically reversible, it is generally accepted that under physiological conditions, the direction of the reaction is optimized to either $\operatorname{NAD}(\mathrm{P})^{+}$ reduction in many photosynthetic organisms, or $\mathrm{NAD}(\mathrm{P}) \mathrm{H}$ oxidation in non-photosynthetic organisms and tissues. Indeed, previous studies on the TrxR-type FNR from the heterotroph Bacillus subtilis ( $B s \mathrm{FNR}$ ) revealed that $B s \mathrm{FNR}$ preferentially oxidizes NADPH to reduce Fd from the same organism at a high rate $\left(\sim 50 \mathrm{~s}^{-1}\right)$, rather than reducing $\mathrm{NADP}^{+}$with electrons from the reduced $\mathrm{Fd}$ (Seo et al. 2004, 2014). Pre-steady state reaction analysis of $B s F N R$ with $\mathrm{NADP}^{+} / \mathrm{NADPH}$ revealed that the hydride transfer rate for NADPH oxidation direction is much larger than that of $\mathrm{NADP}^{+}$reduction direction (Seo et al. 2016). Therefore, research on $C t F N R$ is important because green sulfur bacteria constitute a unique group among photoautotrophic organisms in that they contain TrxR-type FNRs that support NAD $(\mathrm{P})^{+}$photoreduction at a high rate (Seo and Sakurai 2002). Some differences between $C t$ FNR and other TrxR-type FNRs have been revealed from deduced amino acid sequences and crystal structures of TrxR-type FNRs, for example the residue stacking on the re-face of the isoalloxazine ring moiety of the FAD prosthetic group is Phe337 and C-terminal extension region in $C t$ FNR is longer and rich in Lys and Glu residues (Fig. 1a, b) (Muraki et al. 2010; Komori et al. 2010). It is expected that comparative studies of $C t$ FNR with other members of the TrxR-type FNR family will provide valuable information on the structure-function relationships of this family and other flavin-containing NADPH-dependent oxidoreductases. In this work, we performed pre-steady-state kinetic analyses of reactions of $C t$ FNR with $\mathrm{NADP}^{+}$and NADPH. Our studies confirmed that the reaction between $C$. tepidum FNR and $\mathrm{NADP}^{+} / \mathrm{NADPH}$ is reversible as often been reported among plant-type FNR from photosynthetic organisms. 


\section{Materials and Methods}

\section{Preparation of $C$. tepidum FNR}

$C t$ FNR was expressed in $E$. coli cells as described in Muraki et al. (2008). Purification of the $C t$ FNR was performed basically according to the methods used for BsFNR (Seo et al. 2014), except that precipitate obtained by the addition of ammonium sulfate between $35 \%$ and $80 \%$ saturation was used for purification in place of that obtained from $0-40 \%$ saturation.

\section{Stopped-flow spectrophotometry}

Stopped-flow spectrophotometry was performed in a glove box under a nitrogen atmosphere containing hydrogen gas (ca. 5\%) to decrease the residual $\mathrm{O}_{2}(<50 \mathrm{ppm})$ with a palladium catalyst as previously described (Seo et al. 2016). The reaction was initiated by mixing equal volumes of solutions in a single mixing mode at $10^{\circ} \mathrm{C}$. The dead-time of the setup was estimated to be about $1 \mathrm{~ms}$ (Seo et al. 2016). Spectra were collected every 1 ms (Figs. 1-3, 4a-c) or 5 ms (Fig. 4e). All protein and substrate concentrations are given as the final concentrations after mixing unless otherwise stated.

Reduced $C t \mathrm{FNR}$ ( $C t \mathrm{FNR}_{\mathrm{red}}$ ) was obtained using sodium dithionite and methyl viologen as the reductant and redox mediator, respectively, under anaerobic conditions in the glove box. Two milliliters of approximately $200 \mu \mathrm{M} C t$ FNR solution in 20 mM HEPES-NaOH buffer (pH 7.0) were reduced by the addition of an excess of sodium dithionite $(0.4 \mathrm{mg} / \mathrm{ml}$ final concentration) in the presence of $10 \mu \mathrm{M}$ methyl viologen. After a few minutes of incubation at $4^{\circ} \mathrm{C}$, excess dithionite and methyl viologen were removed by size-exclusion chromatography (Bio-Gel P-4 gel, Bio-Rad Laboratories, USA) pre-equilibrated with $20 \mathrm{mM}$ HEPES-NaOH buffer (pH 7.0) containing 0.32 $\mu \mathrm{g} / \mathrm{ml}$ sodium dithionite in the glove box. The concentration of $C t$ FNR in the solution was estimated from the absorbance of air-reoxidized $C t$ FNR.

A deuterated form of NADPH, (4S-2 $\left.{ }^{2} \mathrm{H}\right)-\mathrm{NADPD}$ (referred to hereafter as NADPD), was prepared according to published methods (Viola et al. 1979, Ottolina et al. 1989, Pollock et al. 2001) as previously described (Seo et al. 2016). NADP ${ }^{+}$and NADPH were purchased from Oriental Yeast Co., Ltd.

The values of $A_{460}, A_{590}, \varepsilon_{460}$ and $\varepsilon_{590}$ in Figs. 2b-c, 3a-c and 4c, e were obtained by subtracting $A_{800}$ to compensate for signal drift.

\section{Kinetic data analysis}

Data collection and basic arithmetic operations on the transient spectra obtained from stopped-flow experiments were performed using Unispec (ver. 2.51, Unisoku Co., Ltd.) and Excel (ver. 14 and 15, Microsoft Corporation, Redmond, WA, USA) software. Curve fitting to the transient absorptions at a single wavelength was performed using Igor Pro ver. 6.3 software (Wavemetrics, Portland, OR, USA). 


\section{Miscellaneous methods}

UV-Vis spectra were measured using a double beam spectrophotometer (UV-560, JASCO, Japan). Protein and substrate concentrations were determined from the extinction coefficients: $C t \mathrm{FNR}_{\mathrm{ox}}, \varepsilon_{466}$ $=10.3 \mathrm{mM}^{-1} \mathrm{~cm}^{-1}$ (Seo and Sakurai 2002) and NADPH and NADPD, $\varepsilon_{340}=6.2 \mathrm{mM}^{-1} \mathrm{~cm}^{-1}$. NADP concentration was determined in NADPH form using glucose-6-phosphate dehydrogenase and excess amount of glucose-6-phosphate. 


\section{Results}

\section{Reduction of $C t F R_{0 x}$ by NADPH}

Reduction/oxidation reactions catalyzed by $\mathrm{NAD}(\mathrm{P}) \mathrm{H}$-dependent flavoproteins are generally accompanied by the formation of charge transfer complexes (CTCs), namely FNR ${ }_{0 x}-\mathrm{NADPH}$ (CTC1) and $\mathrm{FNR}_{\mathrm{red}}-\mathrm{NADP}^{+}$(CTC-2) before the hydride transfer step, which exhibit a charge transfer (CT) absorption band in the 500-800 nm region (Scheme 1; Massey et al. 1970; Blankenhorn 1975; Batie and Kamin 1984; Lennon and Williams Jr. 1997; Tejero et al. 2007).

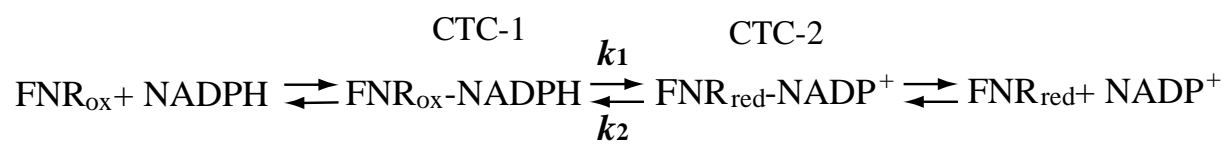

\section{Scheme 1}

The reaction after mixing $9 \mu \mathrm{M} C t \mathrm{FNR}_{\mathrm{ox}}$ with 50-500 $\mu \mathrm{M}$ NADPH was measured by stopped-flow spectrophotometry (Fig. 2). Mixing CtFNR with NADPH resulted in a decrease in flavin absorption band I with a peak around $460 \mathrm{~nm}$ within $1 \mathrm{~ms}$ (at the end of dead time), then the absorption of this band decreased gradually with time (Fig. 2a, b). The $\Delta$ absorbance of this band region contains at least three distinctive kinetic components, namely the fast, slow and very slow phases (Fig. 2c). The fast phase observed as a rapid drop in 0 to $5 \mathrm{~ms}$ was too fast to estimate the rate constant. $\Delta$ absorbance of this phase was almost independent of the NADPH concentrations used (50-500 $\mu \mathrm{M}$, inset of Fig. 2c). $A_{460}$ in 5 to $500 \mathrm{~ms}$ time region can be approximated by a two-step reaction model (Fig. 2c, red continuous lines). $\Delta$ absorbance of the slow phase observed as a decay component in 5-200 ms time region increased with increasing NADPH concentration, and its observed rate decreased with increasing NADPH concentration (Fig. 2d). $k_{\text {obs }}$ of the very slow phase was uncertain as the slight absorbance change seemed to continue beyond the measurement time scale. Although an increase in NADPH concentration decreased the total $\Delta$ absorbance of the flavin absorption band I (Fig. 2a, b), the absorbance change after 499 ms was only $45 \%$ of that expected for fully reduced protein even with $500 \mu \mathrm{M}$ NADPH (Fig. 2b).

The absorbance around $600 \mathrm{~nm}$ rapidly increased within 1 ms and reached its maximum intensity by $5 \mathrm{~ms}$ (Fig. 2a, b). The spectroscopic and kinetic properties of this absorption band centered at 600 $\mathrm{nm}$ are typical of the formation of CTCs as reported in stopped-flow works on $B s F N R$, plant-type FNRs and $E c$ TrxR (Seo et al. 2016; Massey et al. 1970; Batie and Kamin 1984; Tejero et al. 2007; Lennon and Williams Jr. 1997). Subsequently, the absorption intensity of this CT absorption band decreased with time (Fig. 2a, b), but even with $500 \mu \mathrm{M}$ NADPH, about $70 \%$ of the maximum absorption intensity remained after $2 \mathrm{~s}$ (Fig. 2b). No significant peak shift of the CT absorption band was observed within 2 s during $C t \mathrm{FNR}_{\mathrm{ox}}$ reduction by NADPH (Fig. 2a, b). An increase in NADPH 
concentration did not significantly affect the maximum absorption intensity of the CT band, but $\triangle$ absorbance of the decay increased with increasing NADPH concentration (inset of Fig. 2b).

\section{Reduction of $C t F^{2} R_{0 x}$ by NADPD}

We studied the primary kinetic isotope effect by measuring the reactions mixing $11 \mu \mathrm{M} C t \mathrm{FNR}_{\mathrm{ox}}$ with 100 (Fig. 3a), 200 and $500 \mu \mathrm{M}$ (Fig. 3b) NADPD. Mixing $C t \mathrm{FNR}_{\mathrm{ox}}$ with NADPD resulted in a rapid drop in the flavin absorption band I within $1 \mathrm{~ms}$ (Fig. 3a, b), but the $\Delta$ absorbance of the drop after 1 ms was much smaller than that with NADPH (Fig. 2a, b), and the subsequent absorption changes in 0 to 10 ms were slower than those with NADPH. These results indicated that deuterium substitution significantly affected the kinetics of the fast phase in the reduction of $C t \mathrm{FNR}_{\mathrm{ox}}$ (inset of Fig. 3a). The $k_{\text {obs }}$ of the fast phase in the reaction with NADPD was nearly independent of NADPD concentration; its values were $372 \pm 12,434 \pm 11$ and $391 \pm 11 \mathrm{~s}^{-1}$ at 100, 200 and $500 \mu \mathrm{M}$ NADPD, respectively (inset of Fig. 3c). These values contain large errors as they are near the upper limit of the detection of the equipment. Kinetic analyses of the absorbance changes in time region 10 to $1000 \mathrm{~ms}$ provided similar rate constant values for the slow phase in the reaction with NADPH (36 $\pm 8 \mathrm{~s}^{-1}, 22.3 \pm 0.4 \mathrm{~s}^{-1}$ and $19.1 \pm 0.3 \mathrm{~s}^{-1}$ at 100,200 and $500 \mu \mathrm{M}$ NADPD, respectively), which also decreased with increasing NADPD concentration.

The absorption in the CT band region increased rapidly (within $1 \mathrm{~ms}$ ) and reached its maximum within $5 \mathrm{~ms}$ (Fig. 3a, b). Although the reduction of significant amount of FAD prosthetic group occurred in 1-10 ms time period (Fig. 3c), the peak wavelength and intensity of CT band were nearly stable (Fig. 3a, b). Increased NADPD concentration did not significantly affect the maximum intensity of the CT band absorption (Fig. 3a, b). Subsequently, a slight decrease in the absorbance occurred (by $100 \mathrm{~ms}$ ), to a similar extent as was observed in the reaction with NADPH (inset of Fig. 3b). These results, almost the same as those with NADPH, indicate that the formation of CTC-1 was not affected significantly by the deuterium substitution.

\section{Oxidation of CtFNR red by NADP ${ }^{+}$}

$C t \mathrm{FNR}_{\text {red }}$ was prepared with dithionite in the presence of methyl viologen followed by the removal of the latter by size-exclusion chromatography. The spectrum of the reduced $C t$ FNR thus prepared is shown as the thick continuous line in Fig. 4a, b. Mixing $C t$ FNR $_{\text {red }}(12 \mu \mathrm{M})$ with $\mathrm{NADP}^{+}$resulted in a very slow increase in the flavin absorption band I (Fig. 4a, b), indicating that $C t \mathrm{FNR}_{\text {red }}$ oxidation by $\mathrm{NADP}^{+}$was very slow. $A_{460}$ in 0 to 2 sec time region can be approximated by a two-step reaction model (Fig. 4c). The $k_{\text {obs }}$ of the faster component decreased with increasing $\mathrm{NADP}^{+}$concentration (Fig. 4d). The total absorption changes at near equilibrium (i.e. at 2 s) showed a significant dependency on $\mathrm{NADP}^{+}$concentration in the range 100 to $500 \mu \mathrm{M}$ (Fig. 4a, b). From $\Delta A_{460}$ at $2 \mathrm{~s}$, the oxidation level of $C t$ FNR was estimated to be about $70 \%$ (thick broken line in Fig. 4a, b). 
In contrast to the reduction of $C t \mathrm{FNR}_{0 \mathrm{x}}$ by NADPH/NADPD (Figs. 2 and 3), no significant absorption change was observed in the CT absorption band region (550-800 nm) during the reaction of $C t \mathrm{FNR}_{\text {red }}$ with $\mathrm{NADP}^{+}$in the $\mathrm{NADP}^{+}$concentration range 100 to $500 \mu \mathrm{M}$ (Fig. 4a, b).

As the formation of $C t \mathrm{FNR}_{\mathrm{ox}}-\mathrm{NADP}^{+}$complex (Scheme 2) can decrease the amounts of CTCs at the equilibrium when $\mathrm{NADP}^{+}$is excess (Batie and Kamin 1986; Seo et al. 2016), the reaction mixing $C t \mathrm{FNR}_{\text {red }}(24 \mu \mathrm{M})$ with near equimolar $\mathrm{NADP}^{+}(25 \mu \mathrm{M})$ was measured (Fig. 4e). Obtained transient

$$
\mathrm{FNR}_{\mathrm{ox}}+\mathrm{NADP}^{+} \rightleftarrows \mathrm{FNR}_{\mathrm{Ox}} \mathrm{NADP}^{+}
$$

\section{Scheme 2}

spectra exhibited a gradual increase in the CT band absorption with time after 1,000 ms following accumulation of oxidized $C t$ FNR species. $A_{460}$ predicted that the reaction was approximated by a twostep reaction model with similar $k_{\text {obs }}$ in excess NADPH (3.40 $\pm 0.02 \mathrm{~s}^{-1}$ and $\left.0.426 \pm 0.001 \mathrm{~s}^{-1}\right)$ (inset of Fig. 4e). The change of $A_{590}$ can be approximated by two-step reaction model with $k_{\text {obs }}$ of $0.75 \pm$ $0.01 \mathrm{~s}^{-1}$ and $0.123 \pm 0.002 \mathrm{~s}^{-1}$ (inset of Fig. $4 \mathrm{e}$ ). 


\section{Discussion}

Previous pre-steady-state kinetic studies on flavins and other types of flavin-dependent NADP(H) oxidoreductases revealed that mixing with NADPH yielded formations of CTC-1 (FAD ox $-\mathrm{NADPH}$ ) and CTC-2 (FAD red-NADP $^{+}$) with the appearance of CT absorption bands centered at 550-750 nm (Massey et al. 1970; Blankenhorn 1975; Lennon and Williams Jr. 1997; Tejero et al. 2007). The presteady state kinetic study of TrxR-type $B s$ FNR catalyzing reaction with $\mathrm{NADP}^{+} / \mathrm{NADPH}$ revealed that formations of both CTC-1 and CTC-2 exhibited the appearance of CT absorption bands centered at approximately $600 \mathrm{~nm}$ (Seo et al. 2016). In the NADPH oxidation reaction by $C t \mathrm{FNR}_{\mathrm{ox}}$, the CT band centered at approximately $600 \mathrm{~nm}$ appeared within the experimental dead-time. Its maximum intensity was not significantly affected by the NADPH concentration, indicating a formation of Michaelis complex (NADPH-FNR ${ }_{0 x}$, MC-1) occurred before the formation of CTC-1 as frequently reported among flavin-containing NAD(P)H-dependent dehydrogenases (Lennon and Williams Jr. 1997; Tejero et al. 2007) and $100 \mu \mathrm{M}$ NADPH gave near saturation (Seo et al. 2016). After the rapid formation of the CTC-1, reduction of the flavin prosthetic group occurred (Figs. 2a-b, 3a-b). In the reaction, the apparent rate of the fast phase was affected by deuterium substitution (inset of Fig. 3a), indicating that the rate-limiting step in the NADPH oxidation reaction by $C t \mathrm{FNR}_{\mathrm{ox}}$ is the hydride transfer during CTC-2 formation (Scheme 1) in the experimental conditions used. In the course of the reaction, $C t \mathrm{FNR}_{\mathrm{ox}}$ reduction proceeded significantly, while the CT absorption band intensity $\left(A_{590}\right)$ did not change in a corresponding amount. Based on Scheme 1, if CTC-2 has no absorption in the CT band region, a decrease in a CTC-1 amount reduce the absorbance in the CT band region. Thus obtained results could be explained by the presence of a CT absorption band centered at similar wavelength for CTC-2 as observed in BsFNR works (Seo et al. 2016). In the NADPH oxidation reaction by $C t \mathrm{FNR}_{\mathrm{ox}}$, $k_{\text {obs }}$ and $\triangle \mathrm{A}_{460}$ of the slower phase affected by the NADPH concentration (Fig. 2c,d). As the NADPH concentration dependent second order reaction step in Scheme 1 (i.e. formation of MC-1) was rapid,

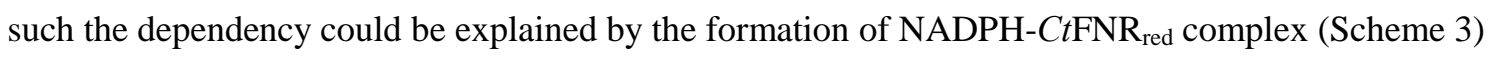
(Batie and Kamin 1986).

$$
\text { FNR red }+ \text { NADPH } \rightleftarrows \text { FNRred_NADPH }
$$

\section{Scheme 3}

For NADPH oxidation by $C t \mathrm{FNR}_{\mathrm{ox}}$, we could not determine the rate constants of the hydride transfer step in both direction ( $k_{1}$ and $k_{2}$ in Scheme 1 ), because the fast phase was too rapid even at 50 $\mu \mathrm{M}$ NADPH (Fig. 2c). If we assume that $C t \mathrm{FNR}_{\mathrm{ox}}$ and CTC-1 have a similar extinction coefficient at $460 \mathrm{~nm}$ (inset of Fig. 2a) and that the hydride transfer step is the rate determining, Scheme 1 can be approximated by the two-component reversible reaction model CTC- $1 \leftrightarrows$ CTC-2, where the rates of the forward and reverse directions corresponds to the respective hydride transfer rates in Scheme 1. According to this approximation, the ratio of CTC-1:CTC-2 at equilibrium at low NADPH concentration (Fig. 1a) corresponds to the ratio of $k_{1}: k_{2}$. From the spectrum at $4 \mathrm{~ms}$ in Fig. 1a, we can 
estimate a CTC-1: CTC-2 ratio of 3:7, suggesting that $k_{2}$ is comparable to $k_{1}$. This result is in contrast to that of $B s F N R$ where the hydride transfer rate in NADPH oxidation direction was much larger than that of $\mathrm{NADP}^{+}$reduction direction ( $\sim 50 \mathrm{~s}^{-1}$ and $<10 \mathrm{~s}^{-1}$, respectively, (Seo et al. 2016)). Similar reversibility has been reported on plant-type FNRs from photosynthetic organisms (Sánchez-Azqueta et al. 2012; Tejero et al. 2007).

Generally, formations of CTCs are considered to lead the stacking of the two-ring system, the isoalloxazine ring and the nicotinamide ring moieties of $\mathrm{FAD}$ and $\mathrm{NAD}(\mathrm{P})^{+} / \mathrm{NAD}(\mathrm{P}) \mathrm{H}$, respectively, which enables efficient hydride transfer between $\mathrm{C} 4$ of nicotinamide ring portion of $\mathrm{NAD}(\mathrm{P})^{+} / \mathrm{NAD}(\mathrm{P}) \mathrm{H}$ and $\mathrm{N} 5$ of isoalloxazine ring portion of FAD in the reaction catalyzed by flavindependent NAD(P)H-oxidoreductases (Blankenhorn 1975; Tejero et al. 2007; Peregrina et al. 2010; Sánchez-Azqueta et al. 2014). The pre-steady state reaction analyses of WT and mutated plant-type Anabaena FNRs with $\mathrm{NADP}^{+} / \mathrm{NADPH}$ revealed a significant correlation between the absorbance of CT bands and hydride transfer rates (Peregrina et al. 2010). In present work, NADPH oxidation by $C t \mathrm{FNR}_{\text {ox }}$ with larger $k_{\text {obs }}$ (Fig. 2) was accompanied by an appearance of a CT absorption band, whereas $\mathrm{NADP}^{+}$reduction reaction with smaller $k_{\text {obs }}$ did no obvious CT absorption band (Fig. 4 ). In the NADP ${ }^{+}$ reduction by $C t \mathrm{FNR}_{\text {red, }}$ as the slope of the initial increasing phase in $A_{460}$ was not affected by the $\mathrm{NADP}^{+}$concentrations used (Fig. 4c), it seemed that the rate of the complex formation with $\mathrm{NADP}^{+}$ was saturated with respect to $\mathrm{NADP}^{+}$concentration. Accordingly, the rate limiting step in $\mathrm{NADP}^{+}$ reduction by $C t \mathrm{FNR}_{\text {red }}$ would be present after the formation of $C t \mathrm{FNR}_{\mathrm{red}}-\mathrm{NADP}^{+}$complex. If the process of $\mathrm{NADP}^{+}$reduction by $C t \mathrm{FNR}_{\text {red }}$ occurred along the same conformations in NADPH oxidation by $C t \mathrm{FNR}_{\mathrm{ox}}$, i.e. kinetically reversible, the hydride transfer process in $\mathrm{NADP}^{+}$reduction by $C t \mathrm{FNR}_{\mathrm{red}}$ would be rapid the same as in the NADPH oxidation by $C t \mathrm{FNR}_{\mathrm{ox}}\left(k_{\mathrm{obs}}=k_{1}+k_{2}\right)$. According to this scenario, the transition of $C t \mathrm{FNR}_{\text {red }}-\mathrm{NADP}^{+}$complex to CTC-2 would be the rate limiting and CTC-2 would not accumulate during the reaction, but the rapid formation of CTC-1 would give an absorption in $\mathrm{CT}$ band region in the $\mathrm{NADP}^{+}$reduction by $C t \mathrm{FNR}_{\text {red. }}$ Obtained results, however, provided almost no $\mathrm{CT}$ absorption bands during the NADP ${ }^{+}$reduction by $C t \mathrm{FNR}_{\text {red. }}$. One can consider that rapid and strong $C t \mathrm{FNR}_{\mathrm{ox}}-\mathrm{NADP}^{+}$complex formation will decrease the absorbance of $\mathrm{CT}$ band region (Scheme 2). But mixing $C t \mathrm{FNR}_{\text {red }}$ with near equimolar of $\mathrm{NADP}^{+}$exhibited a faster formation of $C t \mathrm{FNR}_{\mathrm{ox}}$ followed by a slower formation of CTCs (Fig. 3e). These results seem to indicate a difference in the conformation of CTCs between in the $\mathrm{NADP}^{+}$reduction by $C t \mathrm{FNR}_{\text {red }}$ and in the NADPH oxidation by $C t \mathrm{FNR}_{\mathrm{ox}}$. In the NADPH oxidation reaction, the nicotinamide ring reacts with the oxidized isoalloxazine ring, whereas in the $\mathrm{NADP}^{+}$reduction reaction, the nicotinamide ring reacts with the reduced isoalloxazine ring. The crystal structure analysis of $B s F N R$ with $\mathrm{NADP}^{+}$revealed that the nicotinamide ring portion of $\mathrm{NADP}^{+}$attached on the surface of the NADPH-binding domain with substantial electron density and within a hydrogen-bonding distance to Asp177 (Fig. 1a). In this conformation, $S-{ }^{2} \mathrm{H}$ at $\mathrm{C} 4$ of the nicotinamide moiety of NADPD can face to the N5 of isoalloxazine 
ring portion by the domain motion, which is consistent with the results of the primarily kinetic isotope effect (Seo et al. 2016). Similarly, structurally related $E c$ TrxR also provided a substantial isotope effect in steady-state assay with $S-{ }^{2} \mathrm{H}$ NADPD (Williams Jr. 1995). The crystal structure analyses of $E c \operatorname{TrxR}$ revealed that the isoalloxazine ring portion of the reduced FAD hydroquinone was in a bent conformation whereas that in the oxidized FAD quinone was in a planar one (Lennon et al. 1999), suggesting that a conformational change of the isoalloxazine ring portion of FAD prosthetic group can occur during the redox transition. In the crystal structure of $B s \mathrm{FNR}_{\mathrm{ox}}$ and $C t \mathrm{FNR}_{\mathrm{ox}}$, the isoalloxazine ring portion was in a planer conformation (Komori et al. 2010; Muraki et al. 2010), but we have no structural information about the conformation of isoalloxazine ring portion in the reduced TrxR-type FNR. On the UV-vis spectra, $B s F_{\text {red }}$ had an apparent small absorption band with a peak at $430 \mathrm{~nm}$, which red-shifted in the presence of $\mathrm{NADP}^{+}$(Seo et al. 2016) whereas in this work, $C t \mathrm{FNR}_{\text {red }}$ had only a small shoulder at $410 \mathrm{~nm}$ (Fig. 4a, b). According to the previous reports discussing the relation between the spectroscopic properties and the conformation of the isoalloxazine ring portion of the reduced flavin hydroquinone (Ghisla et al. 1974; Blankenhorn 1975), it is likely that the isoalloxazine ring moiety in $B s \mathrm{FNR}_{\text {red }}$ was in a more planer conformation whereas that in $C t \mathrm{FNR}_{\text {red }}$ was in a bent one (Ghisla et al 1974). Actually BsFNR demonstrated the rapid formation of CTC-2 with an apparent $\mathrm{CT}$ absorption band in the $\mathrm{NADP}^{+}$reduction reaction (Seo et al. 2016), indicating that the conformation of the isoalloxazine ring portion would affect the accessibility and /or distance of the nicotinamide ring portion to the isoalloxazine ring moiety. In addition, the positioning of the Cterminal extension region might play an important role during the reaction as this region hamper the stacking of the rings (Fig. 1a). Further works especially focused on the C-terminal region should be performed to reveal the mechanism of the redox reactions between TrxR-type FNRs and NADP ${ }^{+} / \mathrm{H}$.

Redox equilibrium between $\mathrm{NADP}^{+} / \mathrm{NADPH}$ and $C t \mathrm{FNR}$ shows that $C t \mathrm{FNR}$ can catalyze $\mathrm{NADP}^{+} / \mathrm{NADPH}$ reduction/oxidation reversibly, which is in accordance with previous report (Seo and Sakurai 2002) and its physiological requirements. However, in our in vitro experiments in the absence of $\mathrm{Fd}$, the rate of $\mathrm{NADP}^{+}$reduction was rather slow compared with those by plant-type FNRs (Batie and Kamin 1984; Tejero et al. 2007) and steady-state assay (Seo and Sakurai 2002). In the report by Batie and Kamin (1984), the rate of $\mathrm{NADP}^{+}$reduction was enhanced by the presence of Fd, which led to the conclusion that ternary complex formation is involved in the reaction. The effects of Fd on the kinetics of $C t \mathrm{FNR}$ reactions remain to be investigated. 


\section{Reference}

Aliverti A, Pandini V, Pennati A, de Rosa M, Zanetti G (2008) Structural and functional diversity of ferredoxin-NADP ${ }^{+}$reductases. Arch Biochem Biophys 474:283-291.

Batie CJ, Kamin H (1984) Ferredoxin:NADP ${ }^{+}$oxidoreductase. Equilibria in binary and ternary complexes with NADP ${ }^{+}$and ferredoxin. J Biol Chem 259:8832-8839.

Batie CJ, Kamin H. (1986) Association of ferredoxin-NADP ${ }^{+}$reductase with NADP(H) specificity and oxidation-reduction properties. J Biol Chem 261:11214-11223.

Bianchi V, Reichard P, Eliasson R, Pontis E, Krook M, Jornvall H, Haggard-Ljungquist E (1993) Escherichia coli ferredoxin $\mathrm{NADP}^{+}$reductase: Activation of $E$. coli anaerobic ribonucleotide reduction, cloning of the gene (fpr), and overexpression of the protein. J Bacteriol 175:1590-1595.

Blankenhorn G (1975) Flavin nicotinamide biscoenzymes: models for the interaction between NADH $(\mathrm{NADPH})$ and flavin in flavoenzymes. Reaction rates and physicochemical properties of intermediate species. Eur J Biochem 50:351-356.

Buchanan BB, Arnon DI (1990) A reverse KREBS cycle in photosynthesis: consensus at last. Photosyn Res 24 47-53.

Ceccarelli EA, Arakaki AK, Cortez N, Carrillo N (2004) Functional plasticity and catalytic efficiency in plant and bacterial ferredoxin-NADP(H) reductases. Biochim Biophys Acta 1698:155-165.

Correll CC, Ludwig ML, Bruns CM, Karplus PA (1993) Structural prototypes for an extended family of flavoprotein reductases: comparison of phthalate dioxygenase reductase with ferredoxin reductase and ferredoxin. Prot Sci 2:2112-2133.

Dym O, Eisenberg D (2001) Sequence-structure analysis of FAD-containing proteins. Prot Sci 10:1712-1728.

Ghisla, S., Massey, V., Lhoste, J.-M., Mayhew, SG (1974) Fluorescence and optical characteristics of reduced flavines and flavoproteins. Biochemistry 13: 589-597.

Hügler M, Sievert SM (2011) Beyond the Calvin cycle: Autotrophic carbon fixation in the ocean. Ann Rev Mar Sci 3: 261-289. 
Kanehisa M, Sato Y, Kawashima M, Furumichi M, Tanabe M (2016) KEGG as a reference resource for gene and protein annotation. Nuc Acids Res 44(D1):D457-462. doi: 10.1093/nar/gkv1070.

Kjær B, Scheller, HV (1996) An isolated reaction center complex from the green sulfur bacterium Chlorobium vibrioforme can photoreduce ferredoxin at high rates. Photosyn Res 47:33-39.

Knaff DB, Hirasawa M (1991) Ferredoxin-dependent chloroplast enzymes. Biochim Biophys Acta 1056:93-125.

Komori H, Seo D, Sakurai T, Higuchi Y (2010) Crystal structure analysis of Bacillus subtilis ferredoxin-NADP ${ }^{+}$oxidoreductase and the structural basis for its substrate selectivity. Prot Sci 19:2279-2290.

Lennon BW, Williams Jr CH (1997) Reductive half-reaction of thioredoxin reductase from Escherichia coli. Biochemistry 36: 9464-9477.

Lennon, BW, Williams Jr CH, Ludwig ML (1999) Crystal structure of reduced thioredoxin reductase from Escherichia coli: Structural flexibility in the isoalloxazine ring of the flavin adenine dinucleotide cofactor, Prot Sci 8:2366-2379.

Massey V, Matthews RG, Foust GP, Howell LG, Williams Jr. CH, Zanetti G, Ronchi S (1970) A new intermediate in TPNH-linked flavoproteins. In H. Sund (ed) Pyridine Nucleotide-dependent Dehydrogenases, Springer-Verlag, Berlin, pp. 393-411.

Mandai T, Fujiwara S, Imaoka S (2009) A novel electron transport system for thermostable CYP175A1 from Thermus thermophilus HB27. FEBS J 276:2416-2429.

Medina M, Gómez-Moreno C (2004) Interaction of ferredoxin-NADP ${ }^{+}$reductase with its substrates: optimal interaction for efficient electron transfer. Photosynth Res 79:113-131.

Munro AW, Girvan HM, McLean KJ (2007) Cytochrome P450-redox partner fusion enzymes. Biochim Biophys Acta 1770:345-359.

Muraki N, Seo D, Shiba T, Sakurai T, Kurisu G (2008) Crystallization and preliminary X-ray studies of ferredoxin-NAD $(\mathrm{P})^{+}$reductase from Chlorobium tepidum. Acta Crystallogr Sec F: Struc Biol Cryst 
Com 64:186-189.

Muraki N, Seo D, Shiba T, Sakurai T, Kurisu G (2010) Asymmetric dimeric structure of ferredoxin$\mathrm{NAD}(\mathrm{P})^{+}$oxidoreductase from the green sulfur bacterium Chlorobaculum tepidum: implications for binding ferredoxin and $\mathrm{NADP}^{+}$. J Mol Biol 401:403-414.

Ogawa T, Furusawa T, Nomura R, Seo D, Hosoya-Matsuda N, Sakurai H, Inoue K (2008) SoxAX binding protein, a novel component of the thiosulfate-oxidizing multienzyme system in the green sulfur bacterium Chlorobium tepidum. J Bacteriol 190:6097-6110.

Ottolina G, Riva S, Carrea G, Danieli B, Buckmann AF (1989) Enzymatic synthesis of [4R$\left.{ }^{2} \mathrm{H}\right] \mathrm{NAD}(\mathrm{P}) \mathrm{H}$ and $\left[4 \mathrm{~S}-{ }^{2} \mathrm{H}\right] \mathrm{NAD}(\mathrm{P}) \mathrm{H}$ and determination of the stereospecificity of $7 \alpha$ - and $12 \alpha-$ hydroxysteroid dehydrogenase. Biochim Biophys Acta 998:173-178.

Peregrina JR, Sánchez-Azqueta A, Herguedas B, Martínez-Júlvez M, Medina M (2010) Role of specific residues in coenzyme binding, charge-transfer complex formation, and catalysis in Anabaena ferredoxin NADP+-reductase. Biochim Biophys Acta 1797:1638-1646.

Pettersen EF, Goddard TD, Huang CC, Couch GS, Greenblatt DM, Meng EC, Ferrin TE (2004) UCSF Chimera--a visualization system for exploratory research and analysis. J Comput Chem 25: 1605-1612.

Pollock VV, Barber MJ (2001) Kinetic and mechanistic properties of biotin sulfoxide reductase. Biochemistry 40:1430-1440.

Sakurai H, Ogawa T, Shiga M, Inoue K (2010) Inorganic sulfur oxidizing system in green sulfur bacteria. Photosyn Res 104:163-176.

Sánchez-Azqueta A, Musumeci MA, Martínez-Júlvez M, Ceccarelli EA, Medina M (2012) Structural backgrounds for the formation of a catalytically competent complex with NADP(H) during hydride transfer in ferredoxin-NADP ${ }^{+}$reductases. Biochim Biophys Acta 1817:1063-1071.

Sánchez-Azqueta A, Catalano-Dupuy DL, López-Rivero A, Tondo ML, Orellano EG, Ceccarelli EA, Medina M (2014) Dynamics of the active site architecture in plant-type ferredoxin-NADP ${ }^{+}$reductases catalytic complexes. Biochim Biophys Acta 1837:1730-1738.

Seo D, Tomioka A, Kusumoto N, Kamo M, Enami I, Sakurai H (2001) Purification of ferredoxins and 
their reaction with purified reaction center complex from the green sulfur bacterium Chlorobium tepidum. Biochim Biophy Acta 1503:377-384.

Seo D, Sakurai H (2002) Purification and characterization of ferredoxin-NAD(P) $)^{+}$reductase from the green sulfur bacterium Chlorobium tepidum. Biochim Biophys Acta 1597:123-132.

Seo D, Kamino K, Inoue K, Sakurai H (2004) Purification and characterization of ferredoxin-NADP ${ }^{+}$ reductase encoded by Bacillus subtilis yumC. Arch Microbiol 182:80-89.

Seo D, Okabe S, Yanase M, Kataoka K, Sakurai T (2009) Studies of interaction of homo-dimeric ferredoxin-NAD $(\mathrm{P})^{+}$oxidoreductases of Bacillus subtilis and Rhodopseudomonas palustris, that are closely related to thioredoxin reductases in amino acid sequence, with ferredoxins and pyridine nucleotide coenzymes. Biochim Biophys Acta 1794:594-601.

Seo D, Asano T, Komori H, Sakurai T (2014) Role of the C-terminal extension stacked on the re-face of the isoalloxazine ring moiety of the flavin adenine dinucleotide prosthetic group in ferredoxinNADP $\left.^{+}\right)$oxidoreductase from Bacillus subtilis. Plant Physiol Biochem 81:143-148.

Seo D, Soeta T, Sakurai H, Sétif P, Sakurai T (2016) Pre-steady-state kinetic studies of redox reactions catalysed by Bacillus subtilis ferredoxin-NADP ${ }^{+}$oxidoreductase with $\mathrm{NADP}^{+} / \mathrm{NADPH}$ and ferredoxin. Biochim Biophys Acta 1857:678-687.

Sevrioukova IF, Li H, Poulos TL (2004). Crystal structure of putidaredoxin reductase from Pseudomonas putida, the final structural component of the cytochrome P450cam monooxygenase. $\mathrm{J}$ Mol Biol 336:889-902.

Sétif P (2001) Ferredoxin and flavodoxin reduction by photosystem I. Biochim Biophys Acta 1507:161-179.

Tang KH, Blankenship RE (2010) Both forward and reverse TCA cycles operate in green sulfur bacteria. J Biol Chem 285:35848-35854.

Tejero J, Peregrina JR, Martínez-Júlvez M, Gutiérrez A, Gómez-Moreno C, Scrutton NS, Medina M (2007) Catalytic mechanism of hydride transfer between $\mathrm{NADP}^{+} / \mathrm{H}$ and ferredoxin-NADP ${ }^{+}$reductase from Anabaena PCC 7119. Arch Biochem Biophys 459:79-90. 
Tsukatani Y, Miyamoto R, Itoh S, Oh-oka H (2004) Function of a PscD subunit in a homodimeric reaction center complex of the photosynthetic green sulfur bacterium Chlorobium tepidum studied by insertional gene inactivation: Regulation of energy transfer and ferredoxin-mediated $\mathrm{NADP}^{+}$reduction on the cytoplasmic side. J Biol Chem 279:51122-51130.

Viola RE, Cook PF, Cleland WW (1979) Stereoselective preparation of deuterated reduced nicotinamide adenine nucleotides and substrates by enzymatic synthesis. Anal Biochem 96:334-340.

Wan JT, Jarrett JT (2002) Electron acceptor specificity of ferredoxin (flavodoxin):NADP ${ }^{+}$ oxidoreductase from Escherichia coli. Arch Biochem Biophys 406:116-126.

Waksman G, Krishna TS, Williams Jr CH, Kuriyan J (1994) Crystal structure of Escherichia coli thioredoxin reductase refined at $2 \AA$ resolution. Implications for a large conformational change during catalysis. J Mol Biol 236:800-816.

Williams Jr. CH (1995) Mechanism and structure of thioredoxin reductase from Escherichia coli. FASEB Journal 9:1267-1276.

Yan Z, Nam YW, Fushinobu S, Wakagi T (2014) Sulfolobus tokodaii ST2133 is characterized as a thioredoxin reductase-like ferredoxin:NADP ${ }^{+}$oxidoreductase. Extremophiles 18:99-110.

Ziegler GA, Vonrhein C, Hanukoglu I, Schulz GE (1999) The structure of adrenodoxin reductase of mitochondrial P450 systems: electron transfer for steroid biosynthesis. J Mol Biol 289:981-990. 


\section{Figure legends}

Fig. 1. (a) Close-up view around the isoalloxazine ring moiety of the FAD prosthetic group and $\mathrm{NADP}^{+}$-binding domain in the crystal structure of $C t \mathrm{FNR}$ (PDB code: 3AB1). Main chains of subunit A which includes the FAD prosthetic group, and C-terminal region of subunit B (I327 to A348) in $C t$ FNR are colored in green and purple, respectively. $\mathrm{NADP}^{+}$is superimposed based on the superposition of NADPH-binding domain of $B s$ FNR which binds NADP ${ }^{+}$(Komori et al 2010, PDB code 3LZW) against $C t$ FNR on UCFS Chimera package (Pettersen et al. 2004). Side chains of Tyr57, Asp64 and Asp177 in subunit A, and Phe337 and Ser339 in subunit B are depicted as a ball and stick model. Hydrogen-bondings between Tyr57 and O2' hydroxyl group of ribitol moiety of FAD, Asp64 and N3 of isoalloxazine ring portion, Asp177 and N7 of nicotinamide ring portion of $\mathrm{NADP}^{+}$, and Ser339 and N5 of isoalloxazine ring portion of FAD are depicted as red dotted lines. The figure was prepared using Discovery Studio 4.0 Visualizer (Accelrys Inc., USA). (b) Partially aligned amino acid sequences around the $s i$-face and $r$-face residues in TrxR-type FNRs. The number of the first amino acid residue in each row is indicated at the left. The positions of the Tyr57, Asp64, Phe337 and Ser339 residues in CtFNR sequence are indicated by arrows at upside. bsu_BSU32110: Bacillus subtilis subsp. subtilis str. 168 FNR, rpa_RPA3954: Rhodopseudomonas palustris CGA009 FNR, ttj_TTHA0465: Thermus thermophilus HB27 FNR, cte_CT1512: Chlorobaculum tepidum FNR, cli_Clim_1719: Chlorobium limicola DSM245 FNR.

Fig. 2. (a, b) Transient spectra induced by mixing $9.0 \mu \mathrm{M} C t \mathrm{FNR}_{\mathrm{ox}}$ with a. $100 \mu \mathrm{M}$ or b. $500 \mu \mathrm{M}$ NADPH. The reaction was performed in $20 \mathrm{mM}$ HEPES-NaOH buffer $(\mathrm{pH} 7.0)$ at $10^{\circ} \mathrm{C}$. The spectra at $0 \mathrm{~ms}$ and 2,000 $\mathrm{ms}$ are shown as thin continuous lines. The spectra shown by thin dotted lines from the top to the bottom at $450 \mathrm{~nm}$ correspond to those at 2, 4, 9, 19, 49, 99, 199 and $499 \mathrm{~ms}$, respectively. The spectrum of $C t \mathrm{FNR}_{\mathrm{ox}}$ in the absence of NADPH is shown as a thick continuous line, and that of $C t \mathrm{FNR}_{\text {red }}$ (see Fig. 4) as a thick dashed line, respectively. Arrows indicate the directions of the absorbance change at the respective wavelengths; the dashed parts of the arrows indicate change that occurred within the first data acquisition period (1 ms). The inset in Fig. 2b shows the time course of $A_{590}$ at a. $100 \mu \mathrm{M}$ and b. $500 \mu \mathrm{M}$ NADPH. The data of the inset are the average of five measurements. (c) The time course of $A_{460}$ after mixing $C t \mathrm{FNR}_{\mathrm{ox}}$ with NADPH. The measurement conditions were the same as those in Fig. 2a, except the NADPH concentrations of a: $0 \mu \mathrm{M}$, b: $50 \mu \mathrm{M}$, c: $100 \mu \mathrm{M}$ d: $200 \mu \mathrm{M}$ and e: $500 \mu \mathrm{M}$. The data are an average of four to five measurements. The time course in the short time range is shown in the inset. Double exponential decay curves based on the data for each NADPH concentration in the time range 5 to $500 \mathrm{~ms}$ are indicated by continuous red lines. (d) NADPH concentration dependency of $k_{\text {obs }}$ of the slow phase. The observed rate constant at each NADPH concentration was obtained as shown in Fig. 2c. One standard deviation of each observed rate constant was less than $0.6 \mathrm{~s}^{-1}$. 
Fig. 3. (a, b) Transient spectra induced by mixing $11 \mu \mathrm{M} C t \mathrm{FNR}_{\text {ox }}$ with a. $100 \mu \mathrm{M}$ or b. $500 \mu \mathrm{M}$ NADPD. The reaction was performed in $20 \mathrm{mM}$ HEPES-NaOH buffer $(\mathrm{pH} 7.0)$ at $10^{\circ} \mathrm{C}$. The spectra at $0 \mathrm{~ms}$ and 1,999 $\mathrm{ms}$ are shown as thin continuous lines. The spectra shown by thin dotted lines from the top to the bottom at $450 \mathrm{~nm}$ correspond to those at 2, 4, 9, 19, 49, 99, 199 and 499 ms, respectively. The spectra of $11 \mu \mathrm{M} C t \mathrm{FNR}_{\text {ox }}$ in the absence of NADPD and $C t \mathrm{FNR}_{\text {red }}$ in $20 \mathrm{mM}$ HEPES-NaOH buffer ( $\mathrm{pH}$ 7.0) are shown as a thick continuous line and a thick dashed line, respectively. Arrows indicate the directions of change in absorbance at the respective wavelengths; the dashed parts of arrows indicate change that occurred within the first acquisition period (1 ms). The insets in Fig. 3a and b show time courses of $\varepsilon_{460}$ (Fig. 3a) and $\varepsilon_{590}$ (Fig. 3b) after mixing $C t$ FNR $_{0 x}$ with $100 \mu \mathrm{M}$ NADPH $(\circ)$ and NADPD $(\Delta)$ shown in Fig. 2a and 3a, respectively. The absorbance was normalized by $C t$ FNR concentration. The dotted line in the inset of Fig. 3a represents the absorption of $C t \mathrm{FNR}_{\mathrm{ox}}$. (c) The time course of $A_{460}$ after mixing $C t \mathrm{FNR}_{\mathrm{ox}}$ with NADPD. The measurement conditions were the same as those in Fig. 3a, except the NADPD concentrations of $0 \mu \mathrm{M}$ (closed circle), $100 \mu \mathrm{M}$ (open circle), $200 \mu \mathrm{M}$ (open triangle) and $500 \mu \mathrm{M}$ (open square). The data are an average of four to five measurements. The time course in the short time range is shown in the inset.

Fig. 4. (a, b) Transient spectra induced by mixing chemically reduced $12 \mu \mathrm{M} C t \mathrm{FNR}$ with a. $100 \mu \mathrm{M}$ and b. $500 \mu \mathrm{M} \mathrm{NADP}^{+}$. The reaction was performed in $20 \mathrm{mM}$ HEPES-NaOH buffer ( $\mathrm{pH} \mathrm{7.0)}$ at $10^{\circ} \mathrm{C}$. The spectra of $12 \mu \mathrm{M}$ of $C t \mathrm{FNR}_{\mathrm{ox}}$ and chemically reduced $C t \mathrm{FNR}_{\text {red }}$ in $20 \mathrm{mM}$ HEPES-NaOH buffer (pH 7.0) are represented by thick dashed and thick continuous lines, respectively. Spectra for $0 \mathrm{~ms}$ and 3,999 ms are indicated by thin continuous lines, and for 9, 19, 49, 99, 199, 499, 999 and 1,999 ms, respectively, by thin dotted lines from the bottom to the top at $450 \mathrm{~nm}$. Arrows indicate the direction of change in absorbance. (c) The time course of $A_{460}$ after mixing $C t \mathrm{FNR}_{\text {red }}$ with $\mathrm{NADP}^{+}$. The measurement conditions were the same as those in Fig. 4a, except the $\mathrm{NADP}^{+}$concentrations of a: 0 $\mu \mathrm{M}, \mathrm{b}: 50 \mu \mathrm{M}$, c: $100 \mu \mathrm{M}$ d: $200 \mu \mathrm{M}$ and e: $500 \mu \mathrm{M}$. The data are an average of four to five measurements. The time course in the short time range is shown in the inset. Double exponential decay curves based on the data for each $\mathrm{NADP}^{+}$concentration are indicated by continuous red lines. (d) $\mathrm{NADP}^{+}$concentration dependency of the observed rate of the faster phase. The observed rate constant at each $\mathrm{NADP}^{+}$concentration was obtained as shown in Fig. 4c. One standard deviation of each observed rate constant was less than $0.2 \mathrm{~s}^{-1}$. (e) Transient spectra induced by mixing chemically reduced $24 \mu \mathrm{M} C t$ FNR with $25 \mu \mathrm{M}$ NADP $^{+}$. The reaction was performed in $20 \mathrm{mM}$ HEPES-NaOH buffer (pH 7.0) at $10^{\circ} \mathrm{C}$. The spectra of $24 \mu \mathrm{M}$ of $C t \mathrm{FNR}_{\mathrm{ox}}$ and chemically reduced $C t \mathrm{FNR}_{\text {red }}$ in 20 $\mathrm{mM}$ HEPES-NaOH buffer ( $\mathrm{pH}$ 7.0) are represented by thick dashed and thick continuous lines, respectively. Spectra for $0 \mathrm{~ms}, 1 \mathrm{~s}$ and $20 \mathrm{~s}$ are indicated by thin continuous lines, and for $9 \mathrm{~ms}, 19 \mathrm{~ms}$, $49 \mathrm{~ms}, 99 \mathrm{~ms}, 199 \mathrm{~ms}, 499 \mathrm{~ms}, 2 \mathrm{~s}, 5 \mathrm{~s}$ and $10 \mathrm{~s}$, respectively, by thin dotted lines from the bottom to 
the top at $450 \mathrm{~nm}$. Arrows indicate the direction of change in absorbance. Inset of Fig. 4e represent the time course of $A_{460}(\circ)$ and $A_{590}(\square)$. The data are average of four measurements. Double exponential decay curves based on the data for both wavelengths are indicated by continuous red lines. 


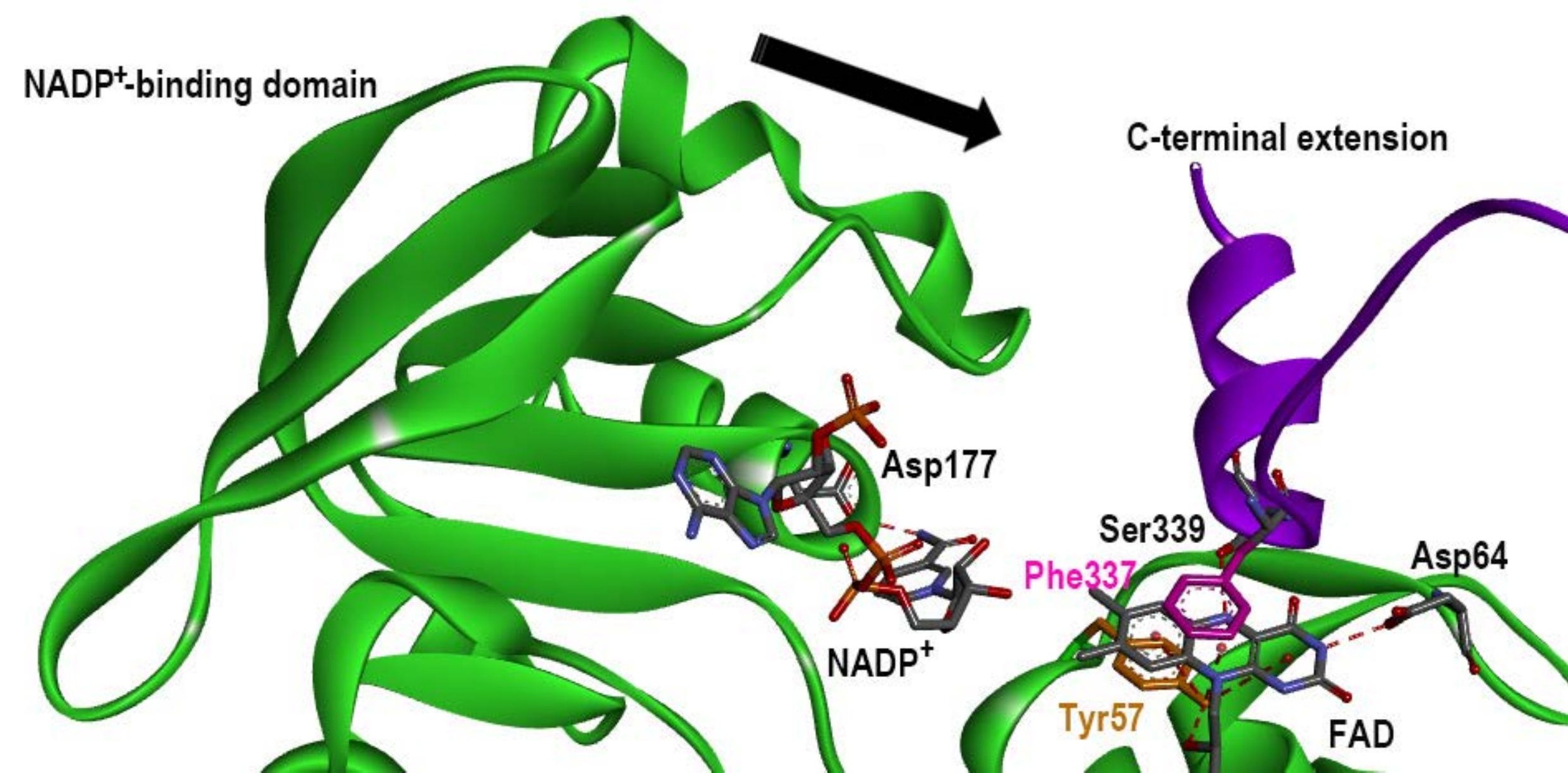




\section{si-face}

bsu_BSU32110 rpa_RPA3954 t七j_TTHA0465 cte_CT1512 cli_Clim_1719

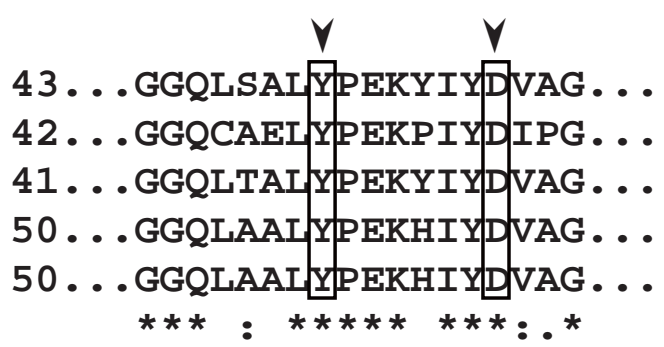

$V \vee$
$313 \ldots$ YMDPKARVQPI角STSLFENK $317 \ldots$ YVYPDKRVVFOY I ISSTNLQKKLGVN 315 . . Y YANPALKVNPGHSSEKAAPGT 326 . . YIKP GEKIRNVF SSVKMAKEKKAAEAGNATENKAE 326 . . YIRPGEKIRHTE SSVKMAKQKKNEQ re-face

bsu_BSU32110 rpa_RPA3954

ttj_TTHA0465

cte_CT1512

cli_Clim_1719
* $*$ : : $:$ : : 
Figure 2a

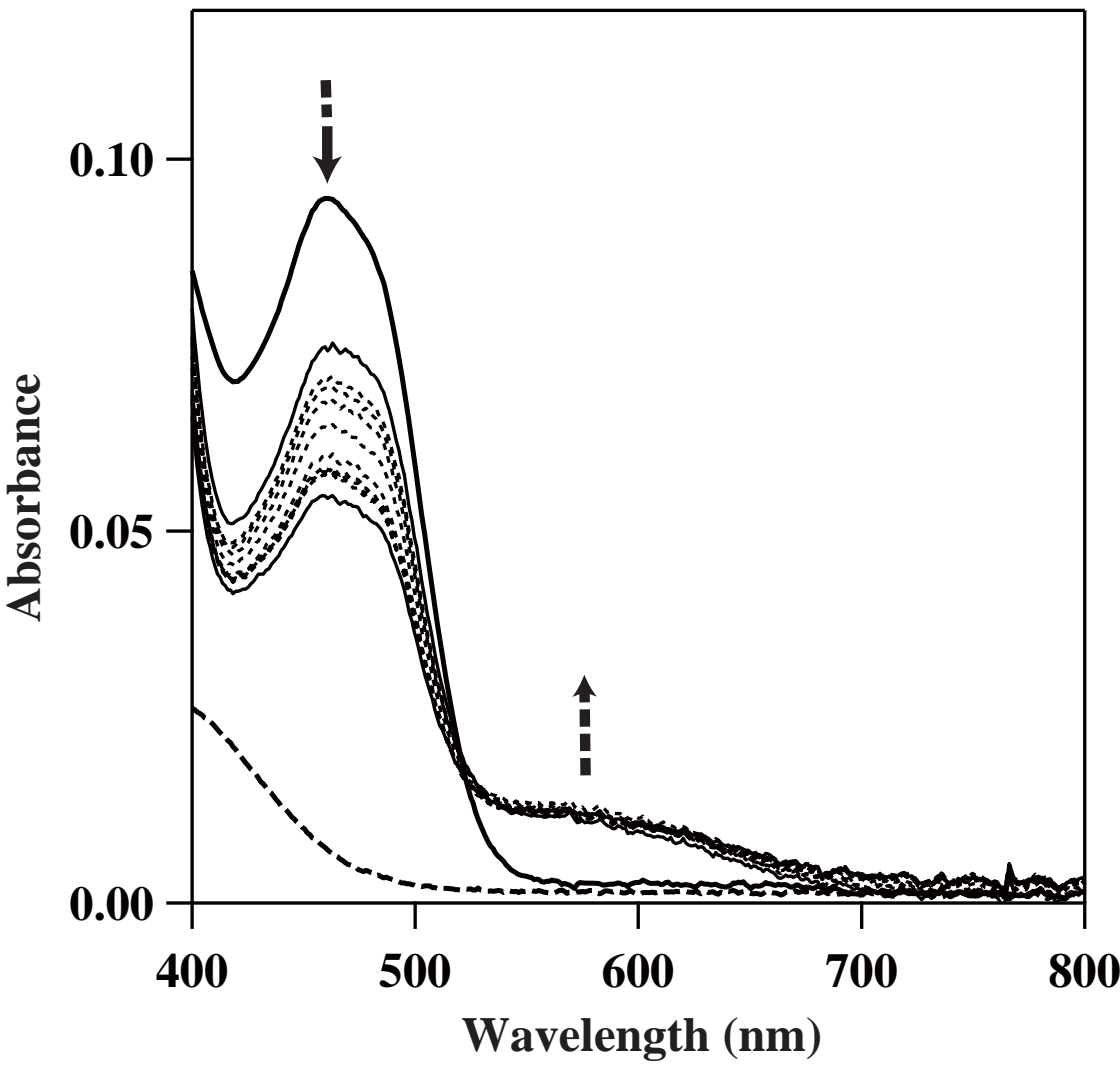


Figure 2b

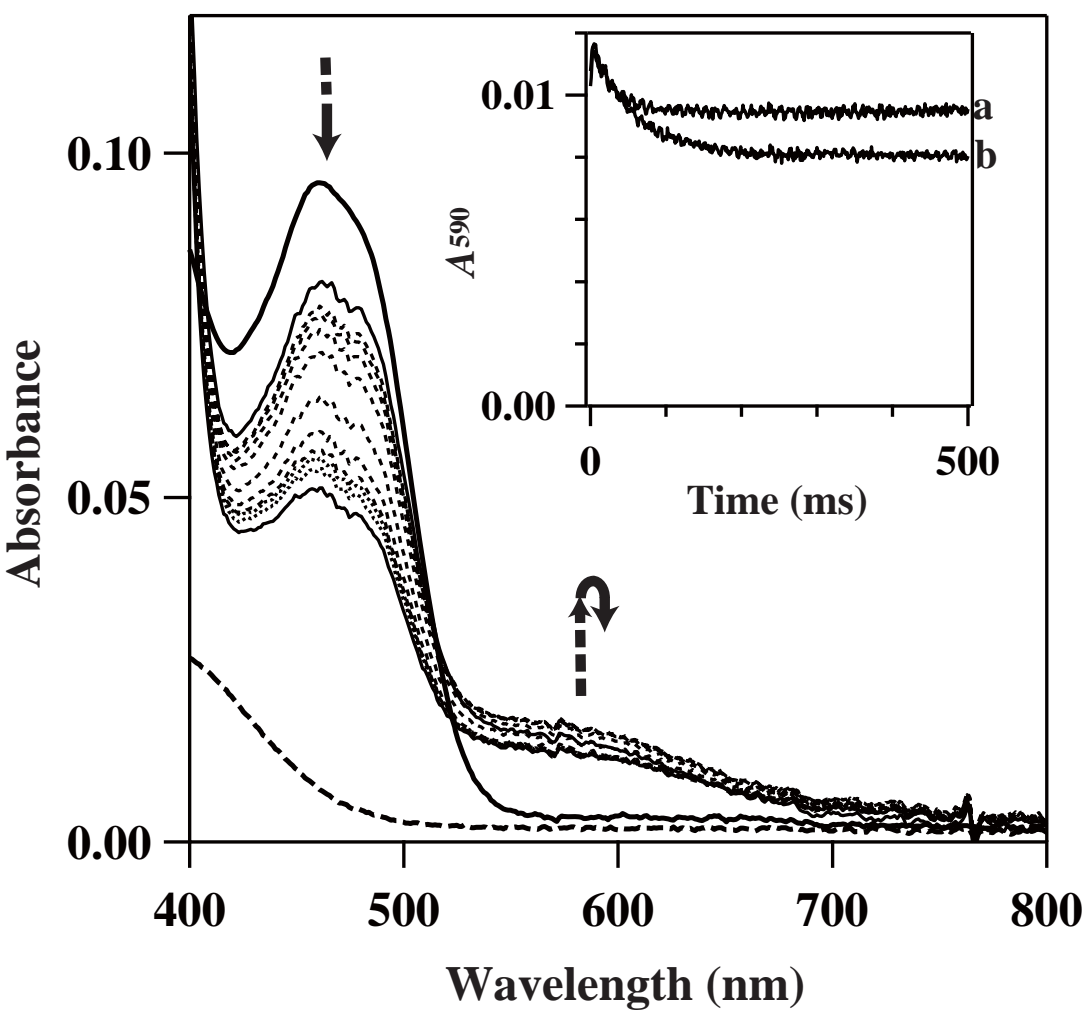


Figure 2c

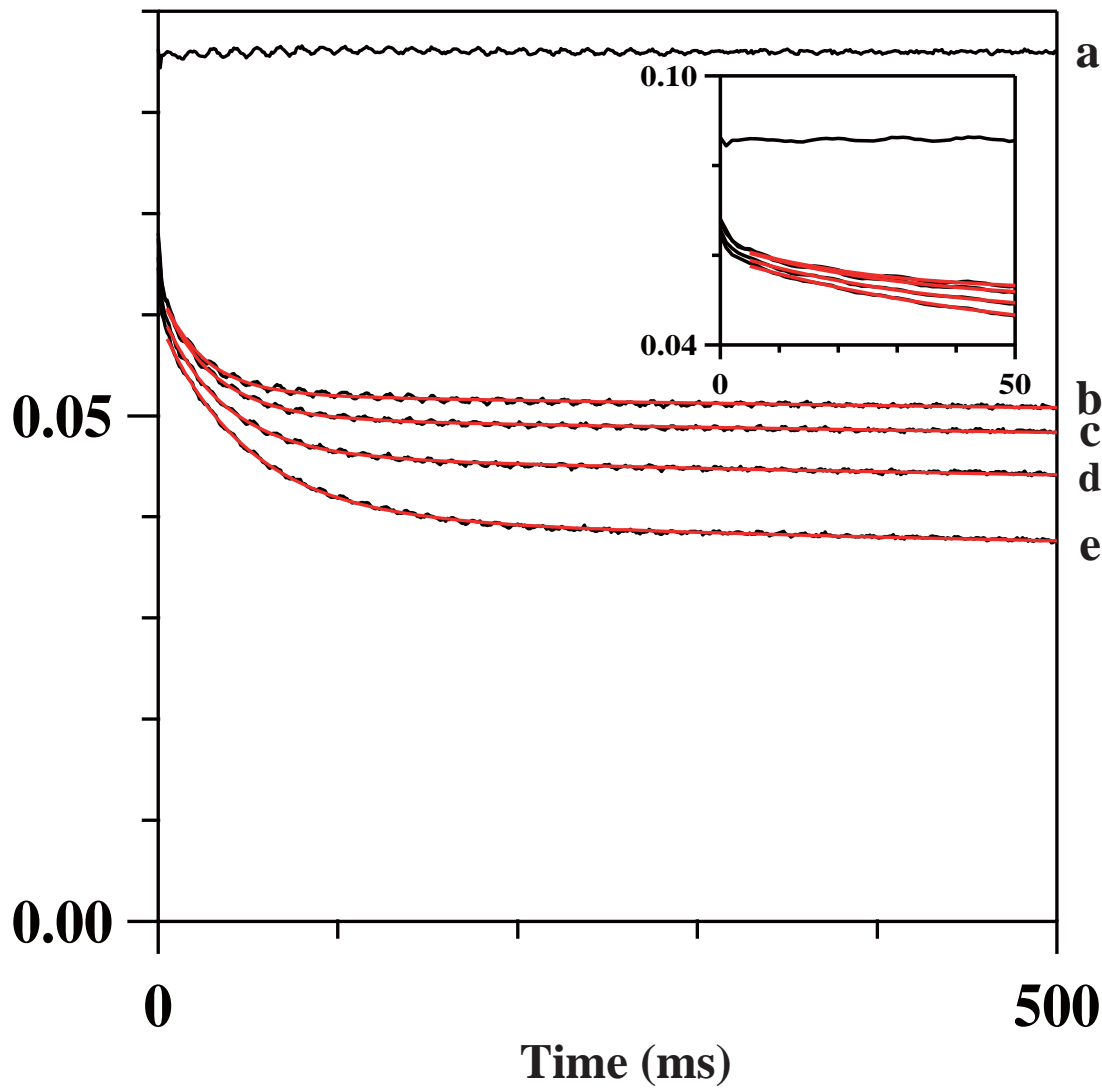


Figure $2 d$

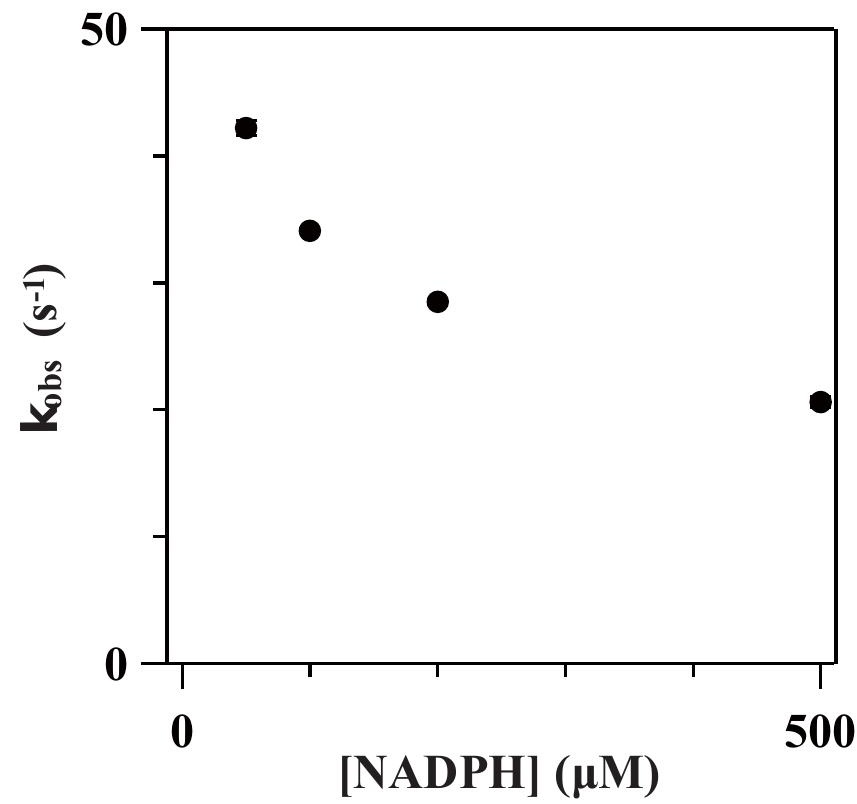


Figure 3a

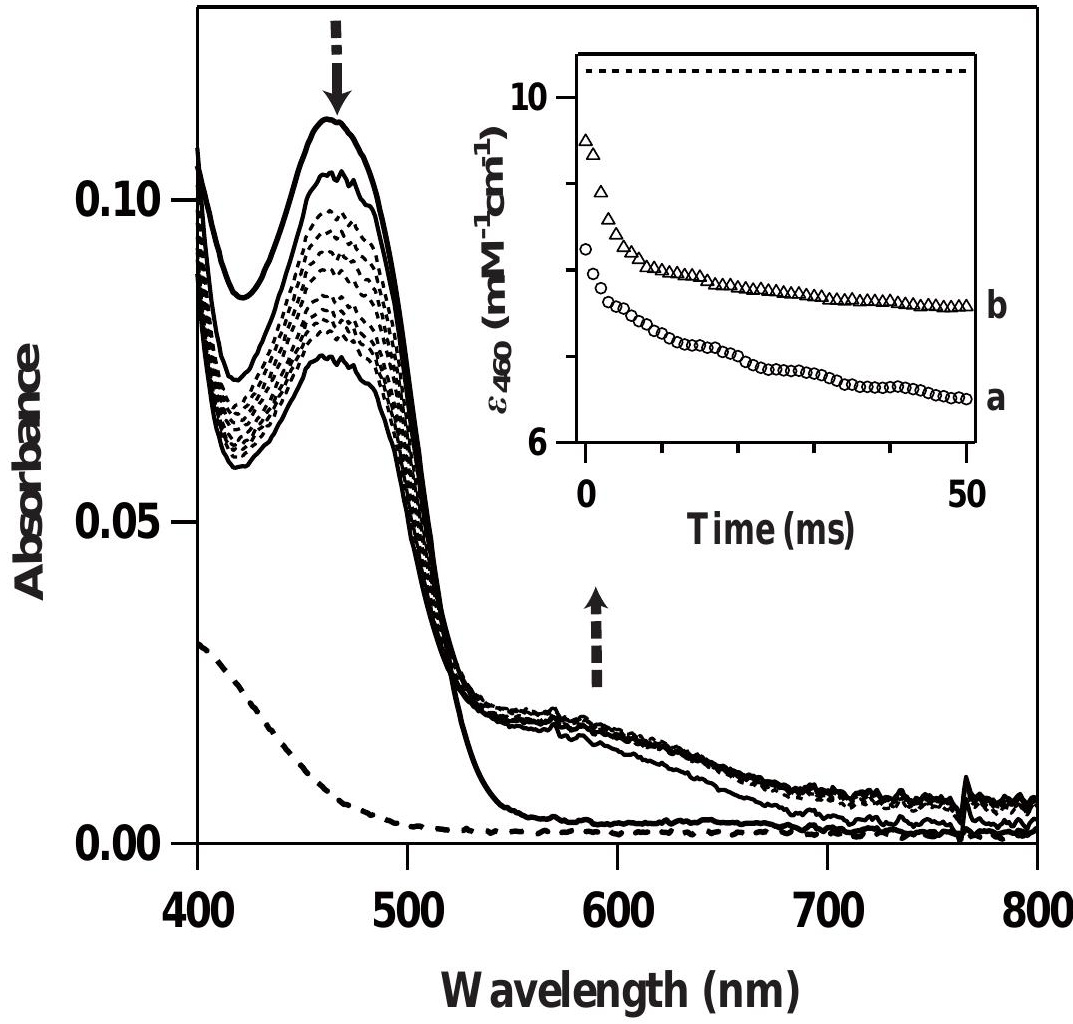




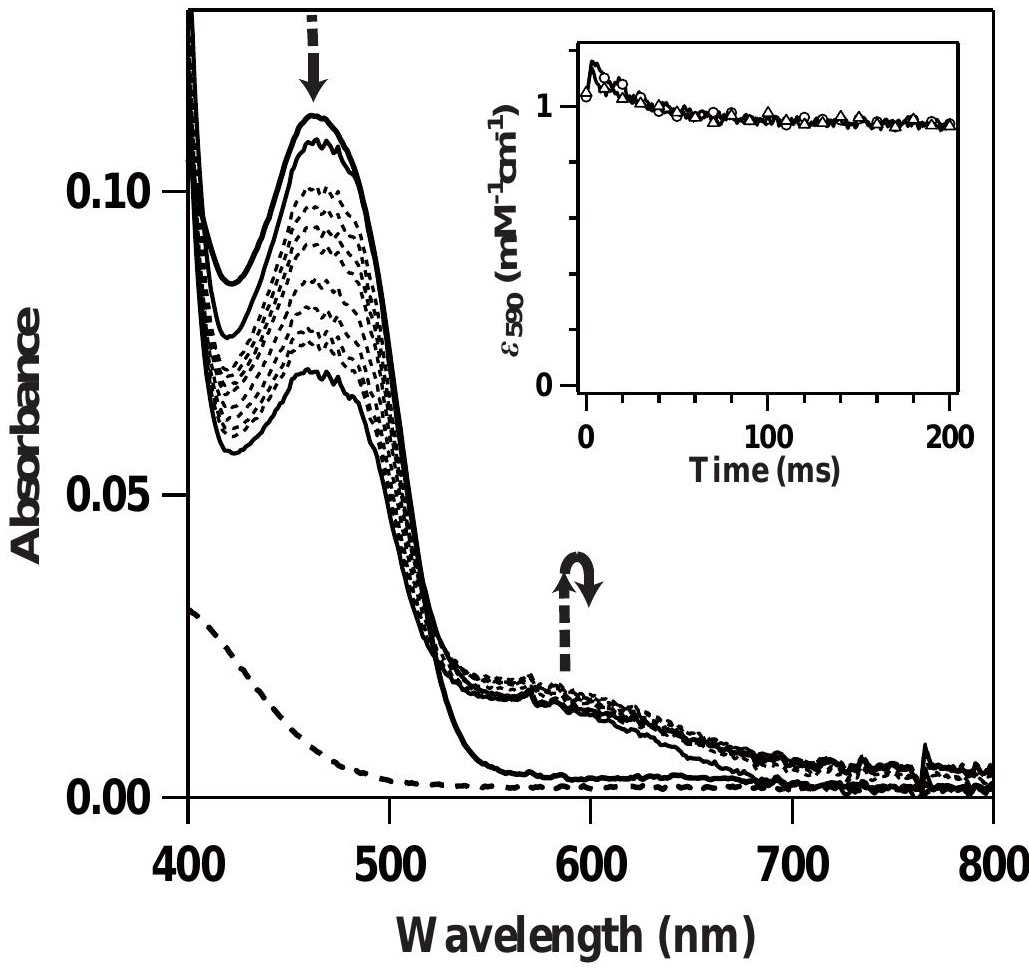




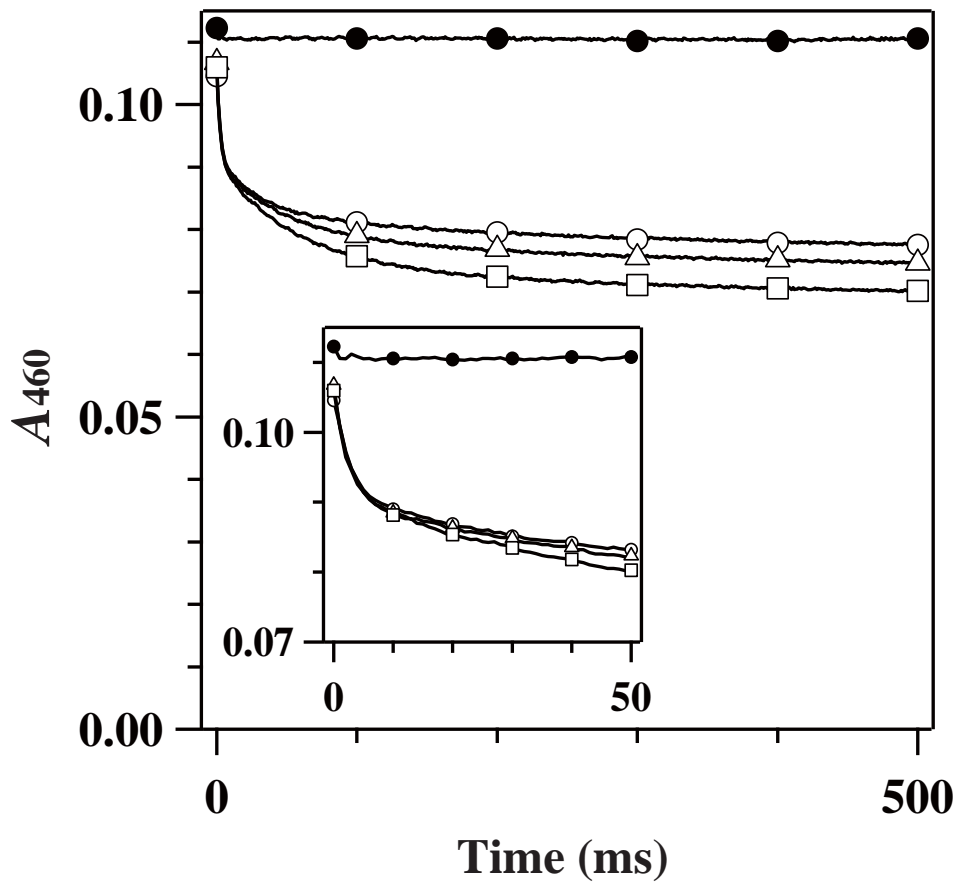


Figure 4a

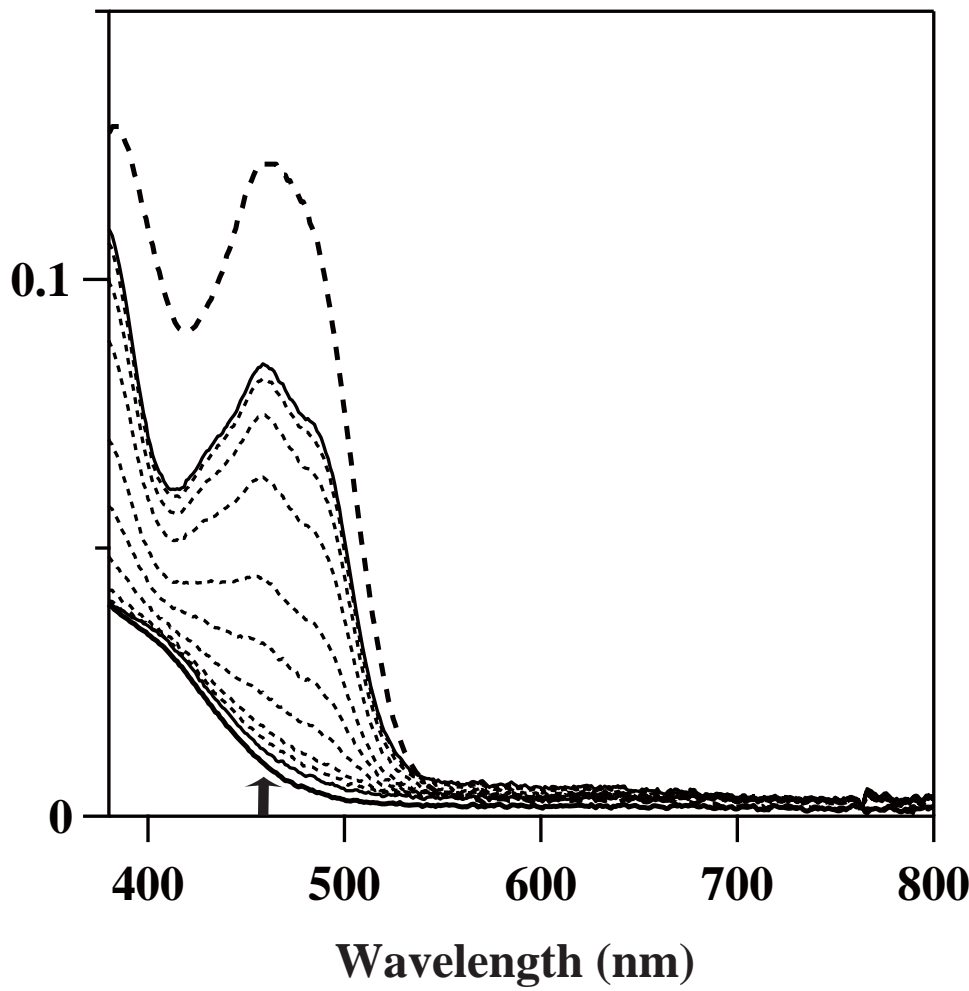


Figure $4 b$

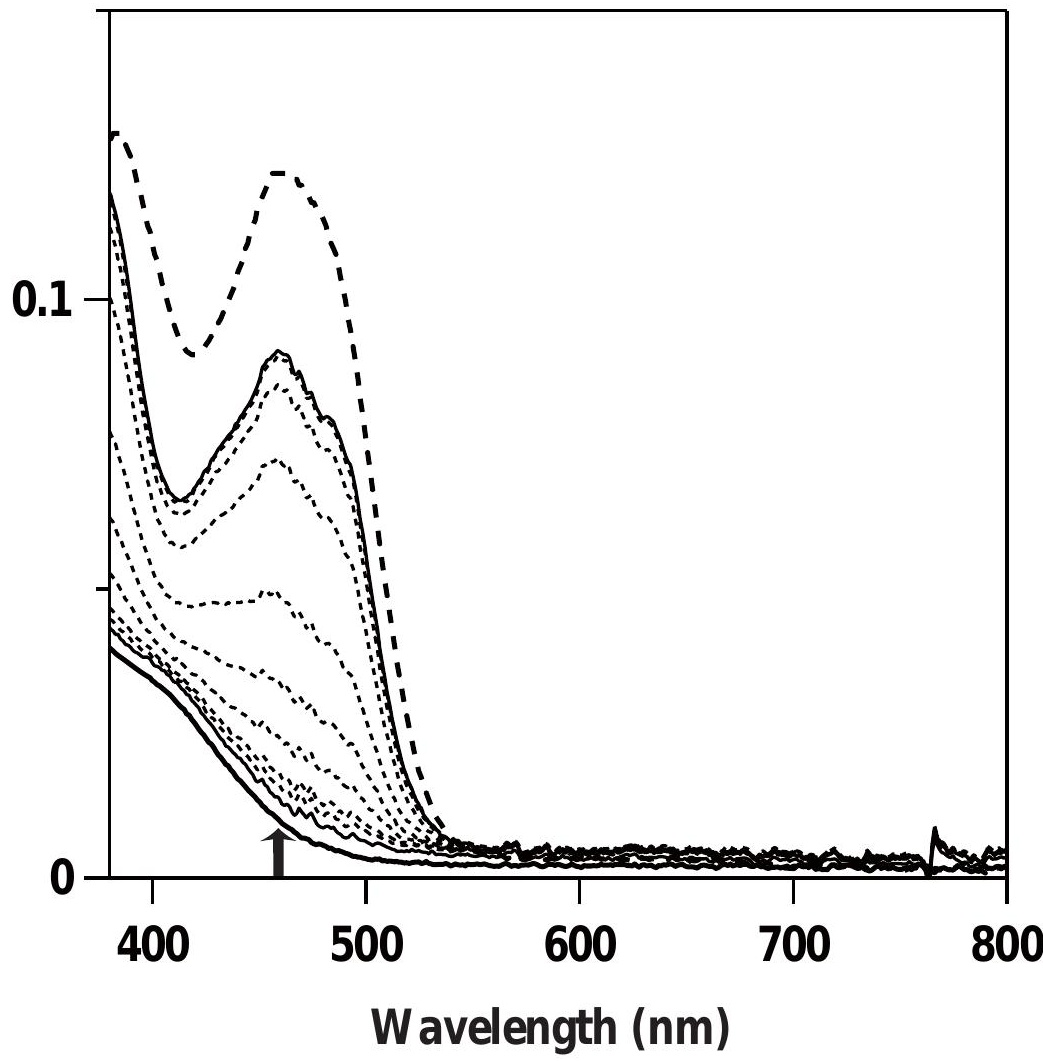


Figure 4c

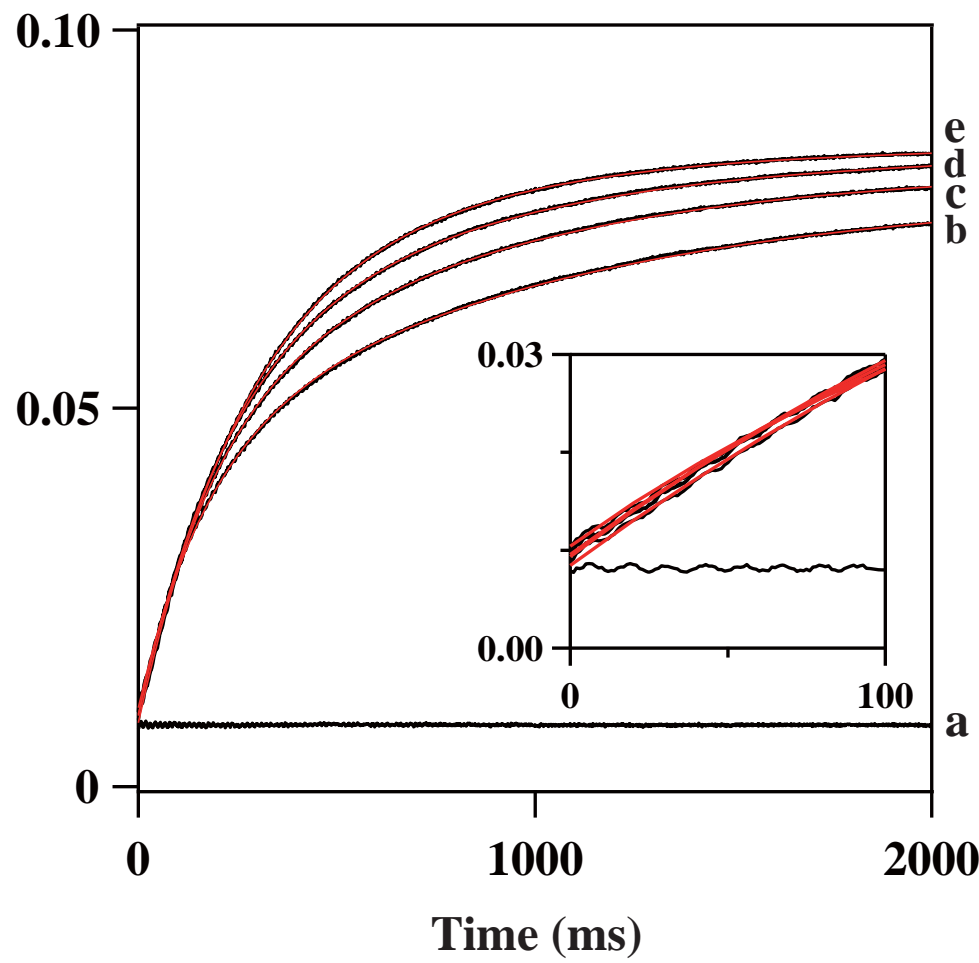




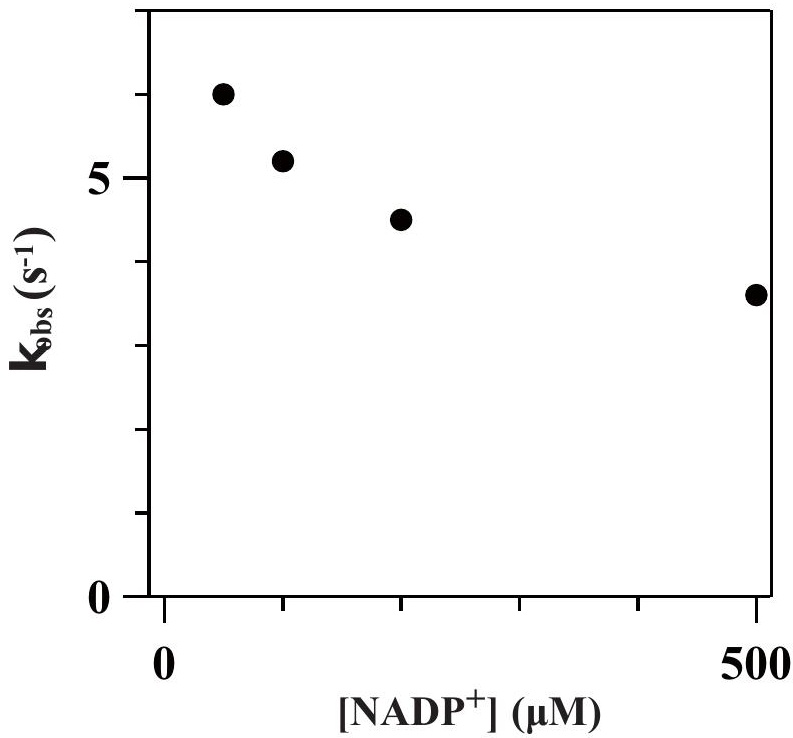




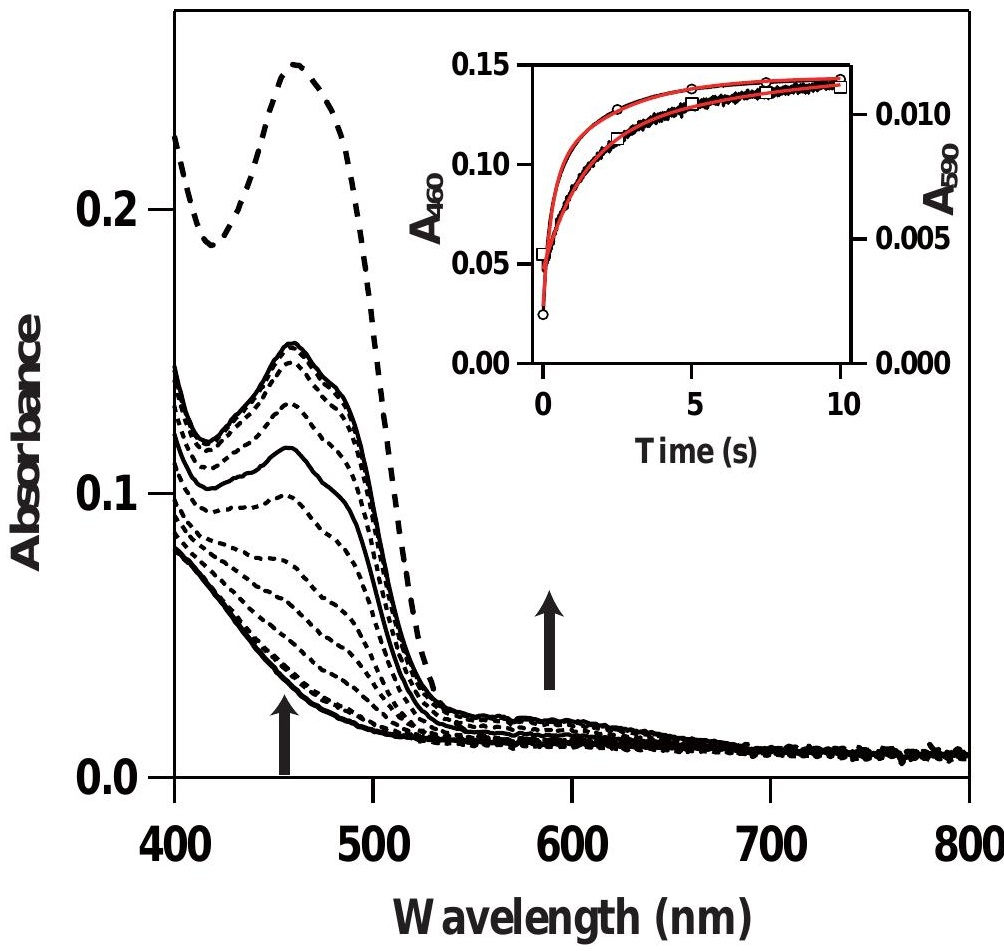

